\title{
Mitochondrial phylogeography and demographic history of the Vicuña: implications for conservation
}

\author{
JC Marín ${ }^{1,2,7}$, CS Casey ${ }^{3,7}, \mathrm{M} \mathrm{Kadwell}{ }^{3}$, $\mathrm{K} \mathrm{Yaya}^{4}$, D Hoces ${ }^{4}$, J Olazabal ${ }^{4,5}$, R Rosadio ${ }^{4,5}$, J Rodriguez ${ }^{6}$, \\ A Spotorno ${ }^{2}$, MW Bruford ${ }^{3}$ and JC Wheeler ${ }^{4}$ \\ ${ }^{1}$ Laboratorio de Genómica y Biodiversidad, Departamento de Ciencias Básicas, Facultad de Ciencias, Universidad del Bio-Bio, Chillán, \\ Chile; ${ }^{2}$ Laboratorio de Genómica Evolutiva de Mamíferos, ICBM, Facultad de Medicina, Universidad de Chile, Santiago, Chile; \\ ${ }^{3}$ Cardiff School of Biosciences, Cardiff University, Cardiff, UK; ${ }^{4}$ CONOPA, Coordinadora de Investigación y Desarrollo de Camélidos \\ Sudamericanos, Lima, Peru; ${ }^{5}$ Facultad de Medicina Veterinaria, Universidad Nacional Mayor de San Marcos, Lima, Peru; \\ ${ }^{6}$ Private Veterinary Practice, Lima, Peru
}

\begin{abstract}
The vicuña (Vicugna vicugna; Miller, 1924) is a conservation success story, having recovered from near extinction in the 1960 s to current population levels estimated at 275000. However, lack of information about its demographic history and genetic diversity has limited both our understanding of its recovery and the development of science-based conservation measures. To examine the evolution and recent demographic history of the vicuña across its current range and to assess its genetic variation and population structure, we sequenced mitochondrial DNA from the control region (CR) for 261 individuals from 29 populations across Peru, Chile and Argentina. Our results suggest that populations currently designated as Vicugna vicugna vicugna and
\end{abstract}

Vicugna vicugna mensalis comprise separate mitochondrial lineages. The current population distribution appears to be the result of a recent demographic expansion associated with the last major glacial event of the Pleistocene in the northern (18 to 22 S) dry Andes 14-12000 years ago and the establishment of an extremely arid belt known as the 'Dry Diagonal' to $29^{\circ} \mathrm{S}$. Within the Dry Diagonal, small populations of $V$. $v$. vicugna appear to have survived showing the genetic signature of demographic isolation, whereas to the north V. v. mensalis populations underwent a rapid demographic expansion before recent anthropogenic impacts.

Heredity (2007) 99, 70-80; doi:10.1038/sj.hdy.6800966; published online 11 April 2007

Keywords: Vicugna vicugna; mtDNA; d-loop; dry diagonal; populations; subspecies

\section{Introduction}

The vicuña (Vicugna vicugna), one of two wild South American camelids, is limited to areas of extreme elevation between $9^{\circ} 30^{\prime}$ and $29^{\prime} \mathrm{S}$ latitude in the Andes. Mitochondrial (mt) DNA sequence data indicate a divergence of at least two million years between vicuña and its wild (and more altitudinally flexible) relative the guanaco (Lama guanicoe; Stanley et al., 1994; Kadwell et al., 2001). Palaeontological evidence suggests that the genus Vicugna evolved from Hemiauchenia in the lowlands east of the Andes as early as two million years ago (Webb, 1974; Harrison, 1979), although a revision of some of this material led Menegaz et al. (1989) to conclude that the vicuña evolved from the guanaco at the beginning of

Correspondence: Dr IC Marín, Laboratorio de Genómica y Biodiversidad, Departamento de Ciencias Básicas, Facultad de Ciencias, Universidad del Bio-Bio, Av. Andrés Bello s/n, Casilla 447, Chillán, Chile.

E-mail:jcmarin@ubiobio.cl

Professor MW Bruford, Cardiff School of Biosciences, Cardiff University, Cardiff, UK.

E-mail: mwbruford@Cardiff.ac.uk

Dr JC Wheeler, CONOPA, Coordinadora de Investigación y Desarrollo de

Camélidos Sudamericanos, Lima, Peru.

E-mail: jwheeler@conopa.org

${ }^{7}$ Joint first authors.

Received 12 October 2006; revised 5 February 2007; accepted 16 February 2007; published online 11 April 2007 the Holocene. Vicuña remains have been found at Tarija, in the Bolivian lowlands (Hoffstetter, 1986), in strata dated to between 97 and 73000 years before present (YBP) (MacFadden et al., 1983) as well as at Cueva Lago Sofia 4 and Tres Arroyos in Tierra del Fuego (Prieto and Canto, 1997) and at Cueva del Medio, Patagonia (Nami and Menegaz, 1991) in archaeological deposits dated to the Pleistocene/Holocene transition approximately 13000 YBP. However, it was only during the last Pleistocene glacial advance in the northern dry Andes (18-22 S) 14-12000 YBP (Ammann et al., 2001; Kull et al., 2002) and the subsequent establishment of the Holocene climatic regime 12-9000 YBP that Vicugna moved into their present high elevation puna habitat (Wheeler et al., 1976; Hoffstetter, 1986). In 1957, Koford (1957) calculated the total Andean vicuña population to be at most 400000 , including 250000 in Peru. However, owing to uncontrolled hunting, by 1969 Grimwood (1969) reported that only 10000 remained in Peru and 2 years later Jungius (1971) estimated a total of 5000-10000 with another 2000 living in Bolivia, Argentina and Chile combined. However, with the introduction of protection and management for sustainable production of its highly prized fine fibre (Wheeler and Hoces Roque, 1997), the vicuña has made a remarkable recovery. Over the last 20 years, it has climbed from endangered status in 1969 to vulnerable in 1972 (Thornback and Jenkins, 1982) to its current population size of $\sim 276000$ (Wheeler, 2006). 
Two subspecies of vicuña are currently accepted, based largely on size differences. Vicugna vicugna vicugna (Molina, 1782) is said to occur between $18^{\circ}$ and $29^{\circ} \mathrm{S}$ and a second, smaller form, Vicugna vicugna mensalis (Thomas, 1917) between $9^{\circ} 30^{\prime}$ and $18^{\circ} \mathrm{S}$. Separation of vicuña subspecies is currently based upon differences in morphology, including the length of molars ( $V . v$. mensalis $45 \mathrm{~mm}, V$. v. vicugna $57 \mathrm{~mm}$ ), height of withers ( $V . v$. mensalis $70 \mathrm{~cm}, V$. v. vicugna $90 \mathrm{~cm}$; Thomas 1917; V. $v$. mensalis $88.5 \mathrm{~cm}$; Wheeler 1995), length of chest hair and pelage color. Most important, the habitat they occupy is fundamentally different. Today, no glaciers exist in the Western Cordillera of the high Andes between $19^{\circ}$ and $27^{\circ} \mathrm{S}$ (Ammann et al., 2001; Kull et al., 2002). This extremely arid belt, referred to as the 'Dry Diagonal', crosses the Andes from NW to SE in the transition zone between the southern hemisphere tropical and westerly wind belt circulation systems, which produce summer precipitation north of $23^{\circ} \mathrm{S}$ and winter precipitation south of $27^{\circ}$, respectively (Ammann et al., 2001; Kull et al., 2002). The Dry Diagonal today receives virtually no precipitation and no glacial formation takes place even on the highest peaks. During the last glaciation of the northern dry Andes (18-22 S) (14000-12 000 years ago), there was increased austral summer precipitation (at least double the present) and depressed snowlines by 700-1000 m (Sharma et al., 1995; Ammann et al., 2001; Kull et al., 2002). South of $23^{\circ}$, precipitation decreased and only weak glacial features are found in the Dry Diagonal to $27^{\circ} \mathrm{S}$. Late Pleistocene glacial records indicate a steeper gradient between the southern glaciers and the Dry Diagonal than exists at present (Ammann et al., 2001; Kull et al., 2002). Climatic changes associated with the last glacial advance in the northern dry Andes led to the formation of massive palaeolakes, most probably without a decrease in temperature (Clayton and Clapperton, 1997; Ammann et al., 2001; Kull et al., 2002) and are thought to have increased the available pasturage for camelids.

Today, vicuña can be divided into those larger populations inhabiting moist puna at high elevations, north of the Dry Diagonal, which loosely conform to the taxon described as $V . v$. mensalis and smaller, relatively isolated populations inhabiting dryer lower elevation puna within the Dry Diagonal (Ammann et al., 2001; Kull et al., 2002), which may correspond to V. v. vicugna. However, the genetic structure of the vicuna along the Andean chain, including the effect of the Dry Diagonal on ecotypic differentiation, has yet to be tested.

To date, our knowledge on the genetics of this species is limited to a few studies. They have documented its phylogenetic relationships and domestication using mtDNA and microsatellite markers (Stanley et al., 1994; Kadwell et al., 2001; Palma et al., 2001), its molecular evolution (Lin et al., 2001; Maté et al., 2004), the variability in allozymes (Norambuena and Paredes, 2003) and the phylogeography and population genetics of wild populations across Chile and Bolivia (Sarno et al., 2004). Here, we present a geographically comprehensive assessment of the molecular diversity of the species, focusing on the mtDNA CR. We aim to reveal aspects of the range-wide phylogeographic structure, including the effect of biogeographical barriers such as the Dry Diagonal and to assess the current taxonomic designations.

\section{Materials and methods}

Two hundred and sixty-one samples were collected between 1994 and 2004 for 18 populations currently designated as $V$. v. mensalis in Peru and from six populations in Chile and five populations in Argentina currently designated as $V$. v. vicugna (Figure 1; Table 1). Samples comprised skin $(n=12)$, blood $(n=246)$ and faeces $(n=3)$. Samples were collected and exported for analysis (CITES permits 6282, 4222, 6007, 5971, 0005177, $0005178,023355,022967$ and 022920$)$ and imported to the UK (permits 269602/01, 262547/02). Total genomic DNA was isolated from blood and skin samples using a standard phenol chloroform extraction method following digestion with proteinase K (Bruford et al., 1998). DNA was precipitated in $100 \%$ ethanol and resuspended in TE buffer $(10 \mathrm{mM}$ Tris- $\mathrm{HCl}, 1 \mathrm{mM}$ ethylenediaminetetraacetic acid, pH 8.0) before analysis. Faecal samples were extracted using the Qiagen DNA stool mini kit (Qiagen Ltd., Crawley, UK) following the manufacturer's instructions. Samples were preserved at $-70^{\circ} \mathrm{C}$ at the Cardiff School of Biosciences, UK; CONOPA, Facultad de Medicina Veterinaria, San Marcos University, Lima, Perú and ICBM, Facultad de Medicina, Universidad de Chile, Santiago, Chile.

The left domain of the mitochondrial CR ( $385 \mathrm{bp}$ ) was amplified using the camelid and vicuña-specific primers LthrArtio (5'-GGT CCT GTA AGC CGA AAA AGG A3'), H15998V (5'-CCA GCT TCA ATT GAT TTG ACT GCG-3'), Loop007V (5'-GTA CTA AAA GAG AAT TTT ATG TC-3'), H362 (5'-GGT TTC ACG CGG CAT GGT GAT T-3') (Marín, 2004). Amplification was performed in $50 \mu \mathrm{l}$ with $\sim 30 \mathrm{ng}$ genomic DNA, $1 \times$ reaction buffer (8 mM Tris- $\mathrm{HCl}$ (pH 8.4), $20 \mathrm{mM} \mathrm{KCl}$ (InvitrogenGibco, Life Technologies, Invitrogen Ltd., Paisley, UK), $2 \mathrm{mM}$ $\mathrm{MgCl}_{2}, 25 \mu \mathrm{M}$ each of deoxyguanosine triphosphate, deoxyadenosine triphosphate, deoxythymidine triphosphate and deoxycytidine triphosphate, $0.5 \mu \mathrm{M}$ each primer and $0.1 \mathrm{U} / \mu$ Taq polymerase (InvitrogenGibco, Life Technologies). Thermocycling conditions were: $95^{\circ} \mathrm{C}$ for $10 \mathrm{~min}$, followed by 30-35 cycles of $94^{\circ} \mathrm{C}$ for $45 \mathrm{~s}, 62^{\circ} \mathrm{C}$ for $45 \mathrm{~s}, 72^{\circ} \mathrm{C}$ for $45 \mathrm{~s}$, then $72^{\circ} \mathrm{C}$ for $5 \mathrm{~min}$. PCR products were purified using the GeneClean Turbo for PCR Kit (Bio101) following the manufacturer's instructions. Products were sequenced in forward and reverse directions using BigDye chemistry on an ABI Prism 377 or 3100 semiautomated DNA analyser. Sequences were trimmed to $328 \mathrm{bp}$ beginning at the $5^{\prime}$ left domain of the d-loop.

SEQUENCHER v.3.02 (Genecodes, Gene Codes Corporation, Ann Arbor, USA) was used to align forward, reverse and consensus sequences and the alignments were rechecked by eye. Genetic variation within populations was first assessed by haplotype $(h)$ and nucleotide diversity $(\pi)$ using Arlequin v.2.000 (Schneider et al., 2000). Molecular variance among populations and countries and between biogeographic regions (ecotype) and subspecies was analysed by nested analysis of molecular variance (AMOVA) using Arlequin v.2.000. Phylogenetic relationships among haplotypes were examined using PAUP v.4.0b10 (Swofford, 2001). The optimal evolutionary model for the data $(\mathrm{HKY}+\mathrm{I}+\mathrm{G}$ corrected, where $-\ln L=711.4193 ; I=0.8250 ; \Gamma=0.8009$; $T_{\mathrm{i}} / T_{\mathrm{v}}$ ratio $\left.=24.748\right)$ was identified using hierarchical likelihood ratio tests using Modeltest v3.06 (Posada and Crandall, 1998). This model provided distance data for 


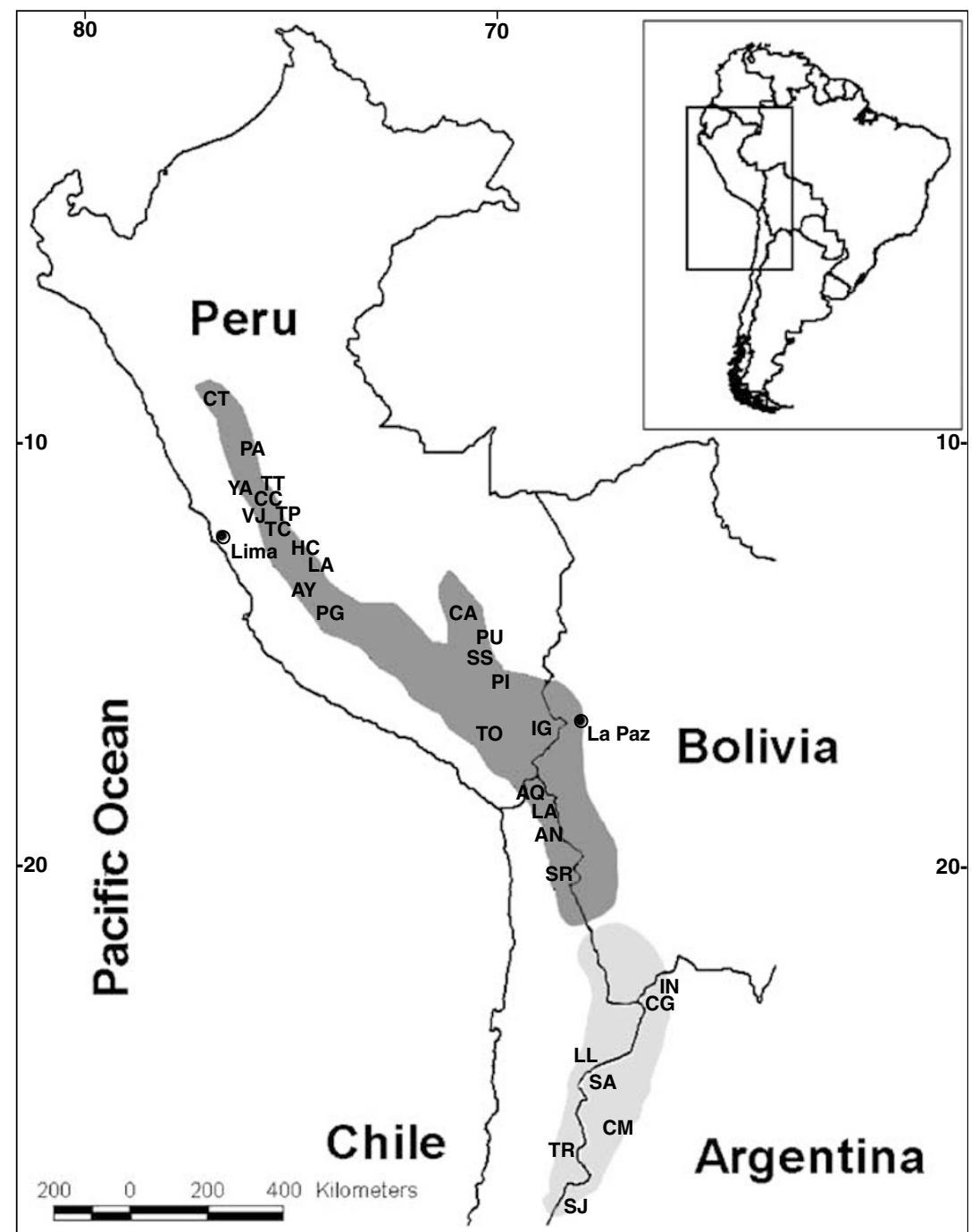

Figure 1 Map of the distribution of Vicugna vicugna mensalis (dark grey) and V. v. vicugna (pale grey) and location of sampled populations. The two letter code indicates the population: CT, Catac; PA, Cerro de Pasco; YA, Yantac; VJ, Villa Junin; TC, Tinco Cancha; TT, Tarma Tambo; TP, Tingo Paccha; CC, Cachi Cachi; AY, Ayavi; PG, Pampa Galeras; HC, Huacarpana; LC, Lachocc; CA, Cerro Azul; SS, Sivina Salma; PU, Puccro; TO, Toccra; PI, Picotani; IG, Ingenio; LA, Lauca; CQ, Caquena; AN, Ankara; SR, Salar Surire; CG, Cineguillas; LL, Llullaillaco; CM, Catamarca; TR, Tres Cruces; SJ, San Juan; IN, Inta; SA, Salta.

neighbour-joining analysis (2000 bootstrap replications). Statistical parsimony networks were constructed. TCS (Clement et al., 2000) was used with a 95\% connection limit to construct the networks for the haplotypes.

Mismatch distributions were produced using Arlequin v.3.000 (Excoffier et al., 2005) to test for evidence of demographic and spatial (demic) expansion (e.g., Ray et al., 2003) and this analysis was augmented using the coalescent-based neutrality estimators, Fu's $F_{S}(\mathrm{Fu}, 1996)$ and Tajima's D (Tajima, 1989), estimated in Arlequin v.2.000 (Schneider et al., 2000) and the point estimator $\theta_{\mathrm{W}}$ (Watterson, 1975) was calculated in DnaSP 3.0 (Rozas and Rozas, 1999). This value was applied as the starting parameter for MCMC simulations in Fluctuate 1.4 (Kuhner et al., 1998) to generate maximum likelihood estimates for $\theta \quad\left(\theta_{\mathrm{g}=\mathrm{var}}\right)$ together with the growth parameter g. Parameter estimation was stabilised by conducting 10 short MCMC chains of 4000 steps each and five long chains of 400000 steps each, with a sampling increment of 20. Three independent runs were conducted.

\section{Results}

Three hundred and twenty-eight base pairs of sequence of the left domain of the mitochondrial CR was generated from 261 samples (Table 2). A total of 28 variable sites, comprising two transversions, 25 transitions and one insertion/deletion defined 34 haplotypes (GenBank Accession numbers AY535255-AY856270). Haplotype $(h)$ and nucleotide diversity $(\pi)$ is detailed in Table 3. Haplotype diversity was found to be high in the species as a whole $(0.7663 \pm 0.0247)$ and also when the data are divided into northern (ostensibly $V . v$. mensalis) and southern ( $V$. v. vicugna) groups $(0.7350 \pm 0.0269$ and $0.9031 \pm 0.0362$, respectively). Overall nucleotide diversity was $0.0119 \pm 0.0066$. Low nucleotide diversity was found in the northern vicuñas $(0.0082 \pm 0.0049)$ 
Table 1 Number of samples analysed from each population of vicuña ( $m$ or v) from Peru, Chile and Argentina with their GPS coordinates

\begin{tabular}{|c|c|c|c|c|c|}
\hline \multirow{2}{*}{$\begin{array}{l}\text { Country } \\
\text { Peru }\end{array}$} & \multirow{2}{*}{$\begin{array}{l}\text { Population } \\
\text { CT: Catac, Ancash }\end{array}$} & \multirow{2}{*}{$\frac{\text { Samples }(\mathrm{n}=261)}{8}$} & \multirow{2}{*}{$\frac{\text { Taxon }}{\mathrm{m}}$} & \multicolumn{2}{|c|}{ Grid Reference } \\
\hline & & & & $090800 \mathrm{~S}$ & $771700 \mathrm{~W}$ \\
\hline & PA: Sta. Ana da Tusi, Pasco & 9 & $\mathrm{~m}$ & $102900 \mathrm{~S}$ & $761900 \mathrm{~W}$ \\
\hline & TC: Tinco Cancha, Junín & 16 & $\mathrm{~m}$ & $110200 \mathrm{~S}$ & $753800 \mathrm{~W}$ \\
\hline & VJ: Villa Junín, Junín & 3 & $\mathrm{~m}$ & $110500 \mathrm{~S}$ & $755200 \mathrm{~W}$ \\
\hline & YA: Yantac, Junín & 18 & $\mathrm{~m}$ & $112000 \mathrm{~S}$ & $761800 \mathrm{~W}$ \\
\hline & TP: Tingo Paccha, Junín & 16 & $\mathrm{~m}$ & $112500 \mathrm{~S}$ & $752700 \mathrm{~W}$ \\
\hline & TT: Tarma Tambo, Junín & 2 & $\mathrm{~m}$ & $113000 \mathrm{~S}$ & $754300 \mathrm{~W}$ \\
\hline & CC: Sto. Domingo de Cachi Cachi, Junín & 4 & $\mathrm{~m}$ & $113800 \mathrm{~s}$ & $753300 \mathrm{~W}$ \\
\hline & HC: San Pedro de Huacarpana, Ica & 17 & $\mathrm{~m}$ & $125000 \mathrm{~S}$ & $750400 \mathrm{~W}$ \\
\hline & LC: Lachocc, Huancavelica & 9 & $\mathrm{~m}$ & $134200 \mathrm{~S}$ & $751500 \mathrm{~W}$ \\
\hline & AY: Ayavi-Tambo-Huaytará, Huancavelica & 5 & $\mathrm{~m}$ & $134200 \mathrm{~S}$ & $751500 \mathrm{~W}$ \\
\hline & PU: Puccro, Cusco & 10 & $\mathrm{~m}$ & $141000 \mathrm{~S}$ & $705700 \mathrm{~W}$ \\
\hline & CA: Cerro Azul, Cusco & 9 & $\mathrm{~m}$ & $141500 \mathrm{~S}$ & $710700 \mathrm{~W}$ \\
\hline & SS: Sivina Salma, Cusco & 9 & $\mathrm{~m}$ & $141500 \mathrm{~S}$ & $705800 \mathrm{~W}$ \\
\hline & PG: R.N.Pampa Galeras, Lucanas, Ayacucho & 41 & $\mathrm{~m}$ & $143900 \mathrm{~S}$ & $752400 \mathrm{~W}$ \\
\hline & PI: S.A.I.S. Picotani, Puno & 6 & $\mathrm{~m}$ & $145500 \mathrm{~S}$ & $700000 \mathrm{~W}$ \\
\hline & TO: Toccra (R.N.S.A.B.), Arequipa & 10 & $\mathrm{~m}$ & $161000 \mathrm{~S}$ & $712000 \mathrm{~W}$ \\
\hline & IG: Ingenio, Huacullani, Puno & 16 & $\mathrm{~m}$ & $164000 \mathrm{~S}$ & $692000 \mathrm{~W}$ \\
\hline \multirow[t]{6}{*}{ Chile } & CQ: Caquena, Putre (I) & 4 & $\mathrm{~m}$ & $181100 \mathrm{~S}$ & $692800 \mathrm{~W}$ \\
\hline & AN: Corral Ankara (I) & 6 & $\mathrm{~m}$ & $182800 \mathrm{~S}$ & $690700 \mathrm{~W}$ \\
\hline & LA: PN Lauca (I) & 11 & $\mathrm{~m}$ & $181200 \mathrm{~S}$ & $691200 \mathrm{~W}$ \\
\hline & SR: Salar de Surire (I) & 5 & $\mathrm{~m}$ & $185500 \mathrm{~S}$ & $690400 \mathrm{~W}$ \\
\hline & LL: PN Lluaillaco (II) & 9 & $\mathrm{v}$ & $244453 \mathrm{~S}$ & $683847 \mathrm{~W}$ \\
\hline & TR: PN Nevado Tres Cruces (III) & 3 & $\mathrm{v}$ & $270529 \mathrm{~S}$ & $685553 \mathrm{~W}$ \\
\hline \multirow[t]{5}{*}{ Argentina } & CG: Cineguillas & 6 & $\mathrm{v}$ & $220600 \mathrm{~S}$ & $655000 \mathrm{~W}$ \\
\hline & IN: INTA Abrapampa & 3 & $\mathrm{v}$ & $222000 \mathrm{~S}$ & $653500 \mathrm{~W}$ \\
\hline & SA: SALTA & 1 & $\mathrm{v}$ & $244249 \mathrm{~S}$ & $685408 \mathrm{~W}$ \\
\hline & CM: Catamarca & 3 & $\mathrm{v}$ & $266500 \mathrm{~S}$ & $660500 \mathrm{~W}$ \\
\hline & SJ: San Juan & 2 & $\mathrm{v}$ & $284000 \mathrm{~S}$ & $673500 \mathrm{~W}$ \\
\hline
\end{tabular}

$\mathrm{m}, V . v$. mensali; $\mathrm{v}, V$. v. vicugna.

A two-letter code is ascribed to each population.

although this was higher for southern populations $(0.0253 \pm 0.0135)$, suggesting that these groups may have contrasting demographic histories. Further subdivisions based on biogeographic region (i.e., potential ecotypes situated within or outside of the Dry Diagonal) supported this supposition. High haplotype diversity coupled with low nucleotide diversity was evident for populations living outside the Dry Diagonal $(0.7540 \pm 0.0257 ; 0.0099 \pm 0.0057$, respectively) with even higher haplotype diversity but also considerably higher nucleotide diversity within it $(0.8758 \pm 0.0610$; $0.0265 \pm 0.0144$, respectively). AMOVA revealed significant $(P<0.001)$ genetic differentiation when populations were grouped by subspecies and biogeographic region (ecotype) and when $V$. v. mensalis populations were grouped by country (but not $V$. v. vicugna). For all analyses, the highest variance component was found within populations (explaining between 51.68 and $98.30 \%$ of the variation, data not shown). However, AMOVA based on subspecies (ecotype) was also significant, accounting for $50.49 \%$ of the variation, as was the analysis based on groups defined by biogeographic region, which accounted for $38.43 \%$ of the variance.

The unrooted NJ tree (Figure 2) using the optimal evolutionary model (see Materials and methods) strongly supported the separation of northern and southern populations. This topology was also consistent with the mtDNA cytochrome $b$ gene phylogeny (not shown). The shallow branches particularly within the northern (mensalis type) group further supported a rapid demographic expansion scenario and little or no branch- ing structure could be detected. On inspection, some degree of geographic structure was apparent in both the tree and network, where haplotypes from adjacent geographic locations generally clustered together.

The network of CR sequences (Figure 3) shows the genealogical relationships among the 34 haplotypes connected through a maximum of 24 mutational steps. A single dominant haplotype (V3) was observed for northern vicuñas from which related sequences were separated by one or two mutational steps. This principal haplotype was shared among populations throughout the northern group, including the range of $V . v$. mensalis, but also included six individuals of the southern type (V. v. vicugna) from Argentina and Chile - all of which were sampled within the dry diagonal. The uniformity of mitochondrial haplotypes across a large geographic range provided further support for a past demographic expansion for the northern populations, but the presence of southern individuals (including $V . v$. vicugna) in this group was unexpected and may be the result of incomplete lineage sorting or, less likely, because of past translocation of animals. Within the network, southern haplotypes were more disparate and none are dominant, a pattern more indicative of small groups surviving in isolated populations.

The mismatch distributions for the northern populations $\left(\tau=5.297 ; \quad \theta_{0}=0.001 ; \quad \theta_{1}=2.827 ; \quad\right.$ SSD $=0.028$ $P=0.47)$ and the whole data set $\left(\tau=13.297 ; \theta_{0}=0.012\right.$; $\theta_{1}=3479$; SSD $=0.024 ; P=0.82$ ) were consistent with a model of a rapidly expanding population, but the southern population result was not $(\tau=11.90$; $\left.\theta_{0}=0.003 ; \theta_{1}=22.588 ; \mathrm{SSD}=0.077 ; P=0.002\right)$. The north- 
Table 2 Alignment of polymorphic sites for 34 vicuna haplotyes obtained for $328 \mathrm{bp}$ of control region sequences

\begin{tabular}{|c|c|c|c|c|c|c|c|c|c|c|c|c|c|c|c|c|c|c|c|c|c|c|c|c|c|c|c|c|c|c|}
\hline Haplotype & 2 & 9 & $\begin{array}{l}1 \\
3\end{array}$ & $\begin{array}{l}1 \\
5\end{array}$ & $\begin{array}{l}1 \\
6\end{array}$ & $\begin{array}{l}1 \\
9\end{array}$ & $\begin{array}{l}2 \\
8\end{array}$ & $\begin{array}{l}2 \\
9\end{array}$ & $\begin{array}{l}3 \\
1\end{array}$ & $\begin{array}{l}3 \\
8\end{array}$ & $\begin{array}{l}5 \\
9\end{array}$ & $\begin{array}{l}6 \\
0\end{array}$ & $\begin{array}{l}6 \\
4\end{array}$ & $\begin{array}{l}6 \\
7\end{array}$ & $\begin{array}{l}6 \\
8\end{array}$ & $\begin{array}{l}6 \\
9\end{array}$ & $\begin{array}{l}8 \\
4\end{array}$ & $\begin{array}{l}1 \\
7 \\
4\end{array}$ & $\begin{array}{l}1 \\
8 \\
1\end{array}$ & $\begin{array}{l}1 \\
9 \\
1\end{array}$ & $\begin{array}{l}1 \\
9 \\
5\end{array}$ & $\begin{array}{l}2 \\
1 \\
1\end{array}$ & $\begin{array}{l}2 \\
1 \\
7\end{array}$ & $\begin{array}{l}2 \\
1 \\
8\end{array}$ & $\begin{array}{l}2 \\
2 \\
9\end{array}$ & $\begin{array}{l}2 \\
6 \\
3\end{array}$ & $\begin{array}{l}2 \\
7 \\
0\end{array}$ & Taxon & Population & $\mathrm{N}$ Total $=261$ \\
\hline V1 & $\mathrm{T}$ & A & $\mathrm{C}$ & G & G & A & $\mathrm{T}$ & $\mathrm{T}$ & A & $\mathrm{C}$ & $\mathrm{T}$ & $\mathrm{T}$ & $\mathrm{T}$ & A & G & $\mathrm{C}$ & A & $\mathrm{T}$ & $\mathrm{C}$ & G & $\mathrm{T}$ & $\mathrm{C}$ & G & $\mathrm{T}$ & $\mathrm{T}$ & $\mathrm{C}$ & $\mathrm{T}$ & $\mathrm{m}$ & PG & 1 \\
\hline V2 & 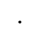 & & . & A & . & . & . & . & . & . & . & . & 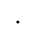 & . & 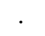 & . & 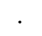 & 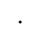 & . & . & . & & A & & . & & . & $\mathrm{m}$ & PG, HC, CC, TO, LA & 14 \\
\hline V3 & . & . & . & A & . & . & . & . & . & . & . & . & . & . & . & . & . & . & . & . & . & . & $\cdot$ & . & . & . & . & $\mathrm{m}, \mathrm{v}$ & $\begin{array}{l}\text { PG, PI, CC, IG, TT, YA, VJ, TC, TP, CA, } \\
\text { SS, PU, TO, LC, CT, PA, LL, CQ, AN, } \\
\text { SR, TR, LA, CM }\end{array}$ & 118 \\
\hline $\mathrm{V} 4$ & . & . & . & . & . & . & . & . & . & . & . & . & . & . & . & . & . & C & . & A & . & $\mathrm{T}$ & . & . & $\mathrm{C}$ & . & . & $\mathrm{m}$ & PG, AY, YA, TO, LC, & 19 \\
\hline V5 & . & . & . & A & . & . & . & . & . & . & . & . & . & . & . & . & . & . & . & A & . & & . & . & . & . & . & $\mathrm{m}$ & PG, IG, CA, SS, PU, TO, LC, AN & 14 \\
\hline V6 & . & . & . & & . & . & . & . & . & . & . & . & . & . & . & . & . & . & . & A & . & $\mathrm{T}$ & . & . & $\mathrm{C}$ & . & . & $\mathrm{m}$ & PG, HC, AY, YA, TC, TO, LC, CT, & 35 \\
\hline V7 & . & . & . & A & . & . & . & . & . & . & . & . & . & . & . & . & . & . & . & A & . & . & . & . & $\mathrm{C}$ & . & . & $\mathrm{m}$ & PG, TO & 3 \\
\hline V8 & . & G & . & 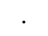 & A & G & $\mathrm{C}$ & $\mathrm{C}$ & . & . & $\mathrm{C}$ & . & $\mathrm{C}$ & G & A & $\mathrm{T}$ & & . & . & A & $\mathrm{C}$ & $\mathrm{T}$ & & . & . & . & . & $\mathrm{v}$ & IN & 2 \\
\hline V9 & . & $\cdot$ & . & & . & . & . & $\mathrm{C}$ & . & . & $\mathrm{C}$ & . & . & . & A & $\mathrm{T}$ & G & . & . & A & . & $\mathrm{T}$ & A & $\mathrm{C}$ & $\mathrm{C}$ & . & . & $\mathrm{v}$ & LL, CG, CM, IN & 6 \\
\hline V10 & . & . & . & . & . & . & . & $\mathrm{C}$ & . & . & $\mathrm{C}$ & . & . & . & A & $\mathrm{T}$ & G & . & . & A & . & . & A & $\mathrm{C}$ & $\mathrm{C}$ & . & . & $\mathrm{m}$ & LA & 2 \\
\hline V11 & . & & . & 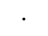 & . & . & . & C & . & . & C & . & . & . & A & $\mathrm{T}$ & G & & . & A & . & $\mathrm{T}$ & A & C & . & . & . & $\mathrm{m}$ & SR, LA & 2 \\
\hline V12 & . & G & . & & A & G & $\mathrm{C}$ & $\mathrm{C}$ & . & . & $\mathrm{C}$ & . & . & . & A & $\mathrm{T}$ & . & $\mathrm{C}$ & . & A & $\mathrm{C}$ & $\mathrm{T}$ & A & . & . & . & . & $\mathrm{m}$ & SR, LA & 2 \\
\hline V13 & . & . & . & A & . & . & . & . & . & . & . & . & . & . & . & . & . & . & . & . & . & . & . & . & $\mathrm{C}$ & . & . & $\mathrm{m}$ & CÁ, SR, LA & 4 \\
\hline V14 & . & G & . & 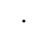 & A & G & $\mathrm{C}$ & $\mathrm{C}$ & . & . & $\mathrm{C}$ & . & & & A & $\mathrm{T}$ & . & C & . & A & $\mathrm{C}$ & $\mathrm{T}$ & A & . & . & $\mathrm{T}$ & . & $\mathrm{m}$ & LA & 1 \\
\hline V15 & . & G & . & . & A & G & C & C & . & . & $\mathrm{C}$ & . & C & G & A & $\mathrm{T}$ & . & C & . & A & C & $\mathrm{T}$ & . & . & C & . & . & $\mathrm{v}$ & SA & 1 \\
\hline V16 & . & . & . & A & . & . & . & . & G & . & . & . & . & . & . & . & . & . & . & . & . & . & A & . & . & . & . & $\mathrm{m}$ & CA & 1 \\
\hline V17 & A & . & . & - & . & . & . & . & . & . & . & . & . & . & . & . & . & . & . & A & . & $\mathrm{T}$ & . & . & $\mathrm{C}$ & . & . & $\mathrm{m}$ & $\mathrm{CT}$ & 1 \\
\hline V18 & . & . & . & . & . & . & . & . & . & . & . & . & . & . & . & . & . & . & . & A & . & . & . & . & $\mathrm{C}$ & . & . & $\mathrm{m}$ & PA & 2 \\
\hline V19 & . & . & . & A & . & . & . & . & . & . & . & . & . & . & . & . & . & . & . & . & . & . & A & $\mathrm{C}$ & . & . & . & $\mathrm{m}$ & HC, YA, VJ, CQ & 9 \\
\hline V20 & . & . & . & . & . & . & . & . & . & . & . & . & . & . & . & . & . & $\mathrm{C}$ & . & A & . & $\mathrm{T}$ & & . & $\mathrm{C}$ & . & $\mathrm{C}$ & $\mathrm{m}$ & PG & 1 \\
\hline V21 & . & . & G & A & . & . & . & . & . & . & . & . & . & . & . & . & . & . & . & . & . & . & . & . & . & . & . & $\mathrm{m}$ & PG & 1 \\
\hline V22 & . & . & . & A & . & . & . & . & . & . & . & $\mathrm{C}$ & . & . & . & . & . & . & . & . & . & & . & . & . & . & . & $\mathrm{m}$ & IG & 5 \\
\hline V23 & . & . & . & . & . & . & . & . & . & . & . & . & . & & . & . & . & . & . & A & . & $\mathrm{T}$ & . & & . & . & . & $\mathrm{m}$ & $\mathrm{HC}$ & 1 \\
\hline V24 & . & . & . & A & . & . & . & . & . & . & . & . & . & . & . & . & . & . & . & . & . & $\mathrm{T}$ & . & . & . & . & . & $\mathrm{m}$ & $\mathrm{TP}$ & 2 \\
\hline V25 & . & 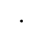 & . & $\cdot$ & . & . & . & C & . & . & C & . & . & . & A & $\mathrm{T}$ & G & C & . & A & . & & A & C & C & . & . & $\mathrm{v}$ & LL & 2 \\
\hline V26 & . & G & . & & A & G & C & C & . & . & $\mathrm{C}$ & . & . & . & A & $\mathrm{T}$ & & . & . & A & C & $\mathrm{T}$ & A & . & . & . & . & $\mathrm{v}$ & LL & 1 \\
\hline V27 & . & G & . & & A & G & C & C & & . & C & . & C & G & A & $\mathrm{T}$ & . & & . & A & C & $\mathrm{T}$ & & . & C & . & . & $\mathrm{v}$ & LL & 2 \\
\hline V28 & . & G & . & & A & G & C & C & . & . & C & . & . & G & A & $\mathrm{T}$ & & C & . & A & C & $\mathrm{T}$ & & . & . & . & . & $\mathrm{v}$ & LL & 1 \\
\hline V29 & . & . & . & A & . & . & . & . & . & . & . & . & . & . & . & . & . & . & $\mathrm{T}$ & . & . & & . & . & C & . & . & $\mathrm{m}$ & CQ & 4 \\
\hline V30 & . & & . & & . & . & . & C & & . & C & . & & & A & $\mathrm{T}$ & & . & . & A & . & $\mathrm{T}$ & A & C & C & & . & $\mathrm{v}$ & TR, SJ & 2 \\
\hline V31 & . & G & . & . & A & G & C & C & . & . & C & . & C & G & A & $\mathrm{T}$ & . & . & . & A & . & $\mathrm{T}$ & . & . & . & . & . & $\mathrm{v}$ & CG & 1 \\
\hline V32 & . & . & . & . & . & . & . & . & . & . & C & . & . & . & A & $\mathrm{T}$ & G & . & . & A & . & . & A & C & . & . & . & $\mathrm{v}$ & CG & 1 \\
\hline V33 & . & & . & A & . & . & . & . & & $\mathrm{T}$ & . & . & & . & . & . & . & . & . & . & . & . & . & . & . & . & . & $\mathrm{v}$ & CG & 1 \\
\hline V34 & . & G & . & . & A & G & C & C & . & . & C & . & C & G & A & $\mathrm{T}$ & . & . & . & A & . & $\mathrm{T}$ & & . & C & . & . & $\mathrm{v}$ & SJ & 1 \\
\hline
\end{tabular}

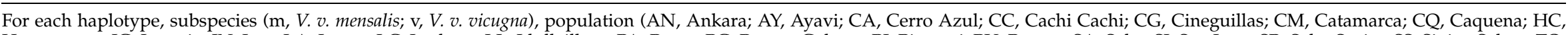
Huacarpana; IG, Ingenio; IN, Inta; LA, Lauca; LC, Lachocc; LL, Llullaillaco; PA, Pasco; PG, Pampa Galeras; PI, Picotani; PU, Puccro; SA, Salta; SJ, San Juan; SR, Salar Surire; SS, Sivina Salma; TO,

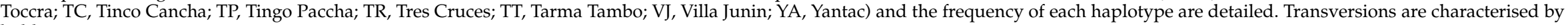
bold type. 
Table 3 Summary of nucleotide diversity $(\pi)$, haplotype diversity $(h)$, and the population growth parameters $\Theta, g$, Fu's $F_{S}$ and Tajima's $D$ for $\mathrm{CR}$ sequences to test the hypotheses of demographic expansion of population groups of vicuna. $\Theta_{\mathrm{w}}$ (Watterson, 1975) was used to estimate $\Theta_{\mathrm{g}=0}$ and to give an initial value for $g$ (Kuhner et al., 1998)

\begin{tabular}{|c|c|c|c|c|c|c|c|}
\hline Population groups & $h \pm$ s.d. & $\pi \pm$ s.d. & $\Theta_{w} \pm s . d$ & $\Theta_{g}=$ var \pm approx s.d. & $g \pm$ approx s.d. & $\mathrm{F}_{\mathrm{S}}$ & $\mathrm{D}$ \\
\hline All populations & $0.7663 \pm 0.0247$ & $0.0119 \pm 0.0066$ & $0.021 . \pm 0.007$ & $0.084 \pm 0.016$ & $262.586 \pm 56.375$ & $-11.125^{* *}$ & $-0.309 \mathrm{NS}$ \\
\hline Inside diagonal & $0.8758+0.0610$ & $0.0265+0.0144$ & $0.021+0.010$ & $0.046+0.014$ & $121.993+47.214$ & 1.349 NS & $2.213 \mathrm{NS}$ \\
\hline Outside diagonal & $0.7540 \pm 0.0257$ & $0.0099 \pm 0.0057$ & $0.022 \pm 0.079$ & $0.069 \pm 0.014$ & $231.104 \pm 56.826$ & $-7.734^{*}$ & -0.754 NS \\
\hline Vicugna & $0.9031 \pm 0.0362$ & $0.0253 \pm 0.0135$ & $0.021 \pm 0.009$ & $0.037 \pm 0.009$ & $99.088 \pm 42.219$ & $-0.086 \mathrm{NS}$ & $2.123 \mathrm{NS}$ \\
\hline Mensalis & $0.7350 \pm 0.0269$ & $0.0082 \pm 0.0049$ & $0.022 \pm 0.008$ & $0.064 \pm 0.014$ & $237.565 \pm 58.460$ & $-5.629 \mathrm{NS}$ & $-0.873 \mathrm{NS}$ \\
\hline Peru & $0.7165 \pm 0.0284$ & $0.0068 \pm 0.0041$ & $0.012 \pm 0.005$ & $0.268 \pm 0.143$ & $1579.847 \pm 287.683$ & -3.705 NS & $0.229 \mathrm{NS}$ \\
\hline Chile & $0.8563 \pm 0.0156$ & $0.0221 \pm 0.0118$ & $0.019 \pm 0.008$ & $0.039 \pm 0.009$ & $109.798 \pm 43.307$ & $-1.124 \mathrm{NS}$ & $1.500 \mathrm{NS}$ \\
\hline Chile mensalis & $0.8369 \pm 0.0751$ & $0.0178 \pm 0.0098$ & $0.021 \pm 0.009$ & $0.029 \pm 0.008$ & $81.846 \pm 46.863$ & $0.544 \mathrm{NS}$ & $0.592 \mathrm{NS}$ \\
\hline Chile vicugna & $0.8788 \pm 0.0751$ & $0.0274 \pm 0.0153$ & $0.012 \pm 0.009$ & $0.034 \pm 0.014$ & $167.669 \pm 71.516$ & $1.535 \mathrm{NS}$ & $1.863 \mathrm{NS}$ \\
\hline Argentina & $0.8857 \pm 0.0686$ & $0.0246 \pm 0.0136$ & $0.022 \pm 0.011$ & $0.030 \pm 0.009$ & $72.355 \pm 43.398$ & $0.286 \mathrm{NS}$ & $1.269 \mathrm{NS}$ \\
\hline
\end{tabular}

Abbreviations: CR, control region; NS, not significant; s.d., standard deviation.

$* * P<0.01, * P<0.05$, NS $P>0.05$.

These values were then used to estimate $\Theta_{g}=$ var and $g$ and their standard deviations, calculated from the two-dimensional likelihood curve of the joint estimates of $\Theta_{\mathrm{g}=\text { var }}$ and $g$.

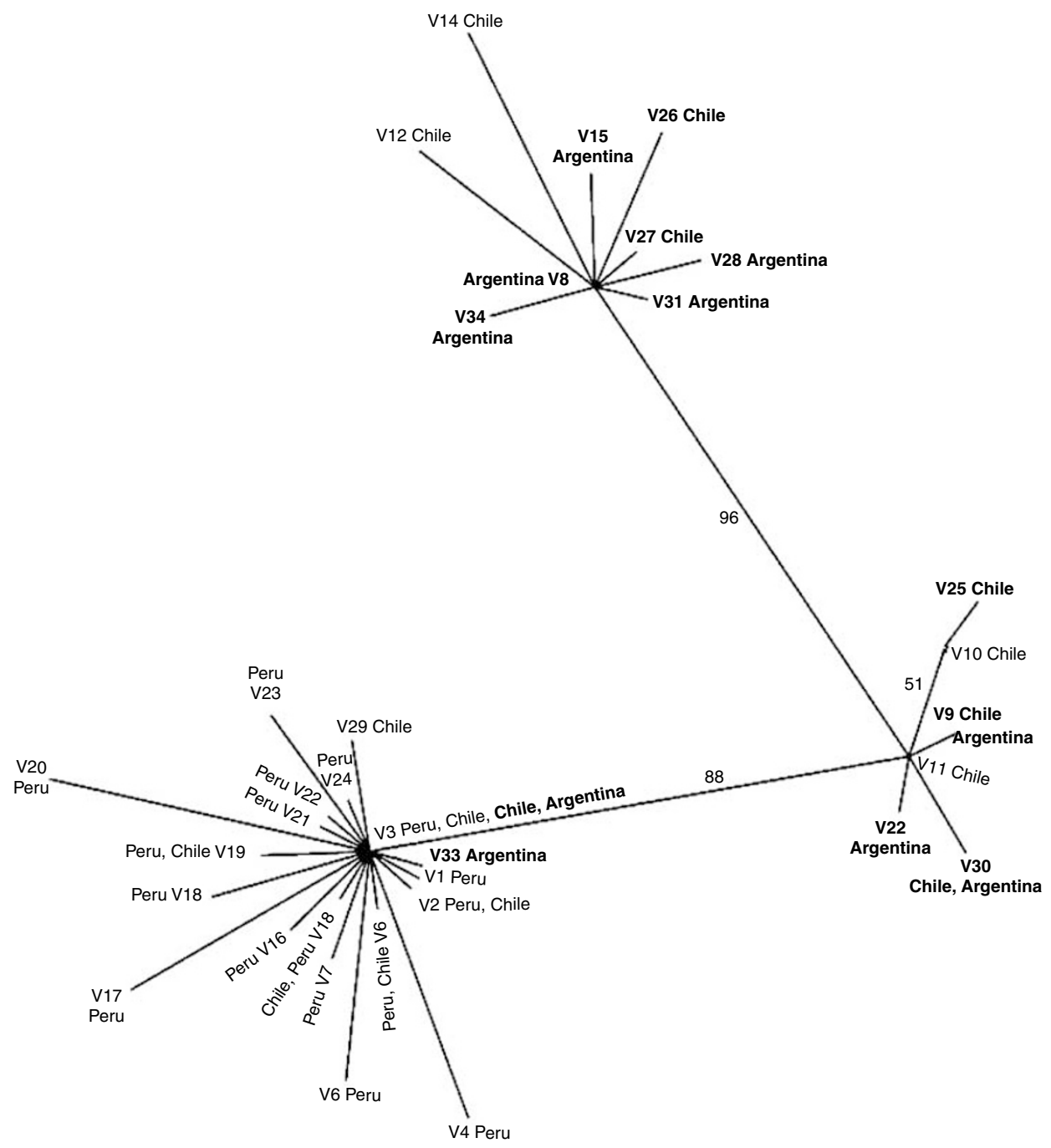

1 change

Figure 2 Neighbour joining phenogram of vicuña mitochondrial CR haplotypes constructed using HKY $+\mathrm{I}+\mathrm{G}$-corrected distances with 2000 bootstrap iterations. Bold type indicates V. v. vicugna and normal type V. v. mensalis.

ern population, however, showed a multimodal pattern which was not expected with sudden expansion from a non-subdivided population, rather with spatial diffusion between adjacent demes (Ray et al., 2003) and yielded the parameters for this model (Excoffier, 2004) as $\tau=2.918$; $\theta=1.796 ; \mathrm{M}=0.931$. 


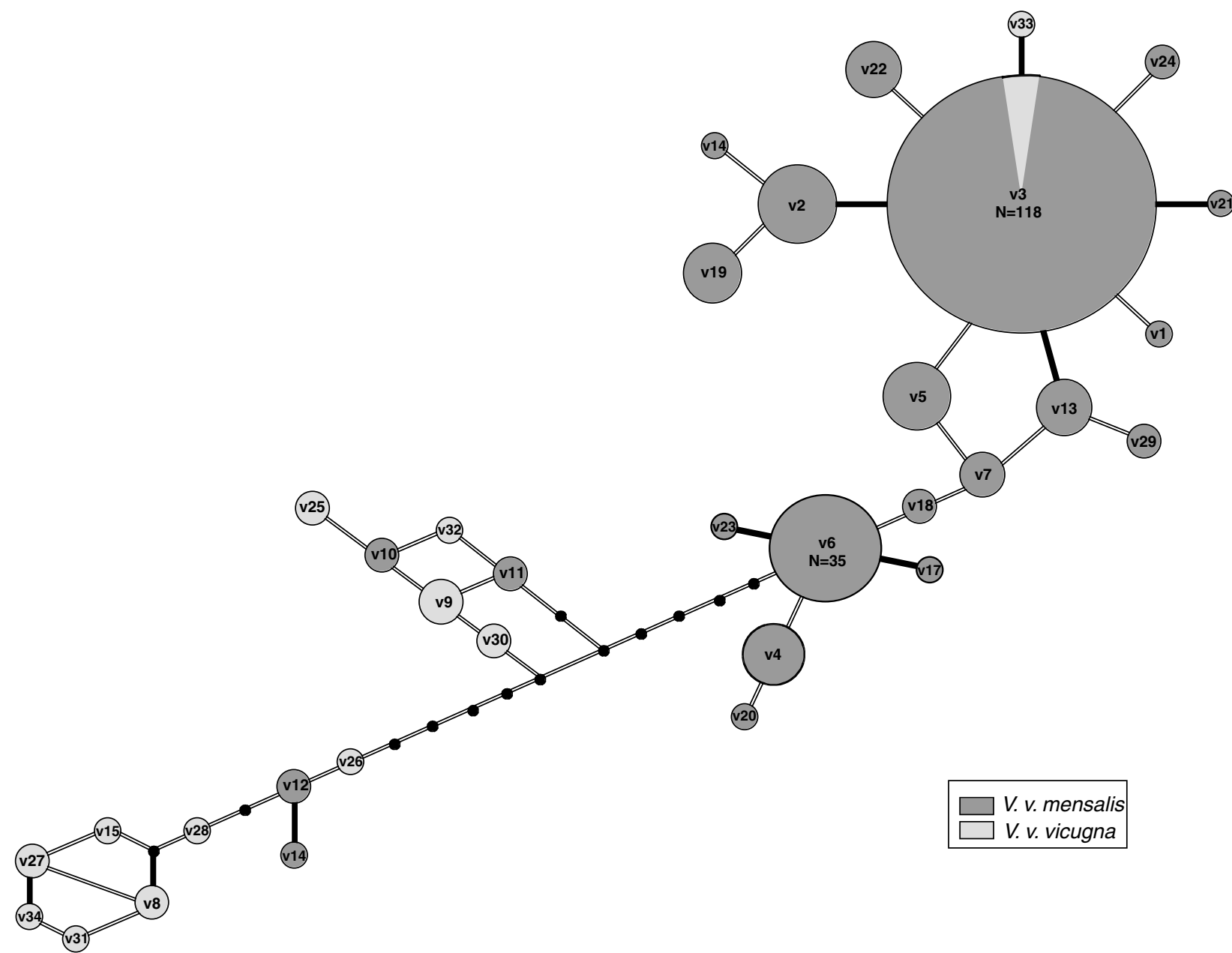

Figure 3 Median joining network of vicuna haplotypes. The size of haplotypes is approximately proportional to their frequency.

The point estimators $\pi$ and $\theta_{\mathrm{w}}$ were similar across all population groups, except for northern populations as a whole and those outside the Dry Diagonal and Peru (which only contains $V . v$. mensalis sequences), where $\pi$ were considerably lower than for other groups. This suggests that only these populations have changed substantially in size over time (Table 3 ). The population growth parameter $(g)$ strongly supported historic population expansion, not only of the whole data set, but also of subpopulation groupings based on biogeographic region, subspecies or country. However, it was clear that the most significant expansion was in the Peruvian populations. In addition, Fu's test for neutrality indicates population expansion for the complete data set $\left(F_{S}=-11.13, P=0.004\right)$ and for the populations outside the Dry Diagonal $\left(F_{S}=-7.734, P=0.05\right)$. Negative $F_{S}$ values were also obtained for northern and southern groupings, but these values were not significant. No population groups appear to show signatures of demographic contraction.

\section{Discussion}

\section{Genetic diversity and phylogeography}

The vicuña as a whole displays a high degree of mitochondrial diversity at the haplotype level with
34 haplotypes from $328 \mathrm{bp}$ of CR sequence $(h=0$, $7663 \pm 0.0247)$ and displays a correspondingly low nucleotide diversity $(0.0119 \pm 0.0066)$. The same pattern of diversity is evident for subspecies, countries and biogeographic regions (ecotypes). Further examination of these results reveals that the southern populations (and those within the Dry Diagonal) are more diverse than those in the north (Figure 4). The lower diversity in northern populations, coupled with the presence of a dominant and widespread haplotype indicates that the northern populations have undergone a rapid demographic expansion. However, the population history of southern vicuna appears different. The levels of diversity in vicuña are in general comparable with those in other montane ungulates (chamois $h=0.000-0.705, \pi=0.000-$ 0.1571; Schaschl et al., 2003; Mongolian sheep $\pi=0.01-$ 0.03; Tserenbataa et al., 2004). In comparison with other taxa, these results broadly agree with those of Sarno et al. (2004) (V. v. mensalis $h=0.110-0.395, \pi=0.002-0.002$ and V. v. vicugna $h=0.209-0.524, \pi=0.005-0.008)$, but when we compare the results for vicuña, our data show higher average levels of both haplotype and nucleotide diversity, except within Chile where the results are similar. This could be because of the larger number of samples included in our study. However, both data sets report higher levels of diversity within $V$. v. vicugna compared with V.v. mensalis. 


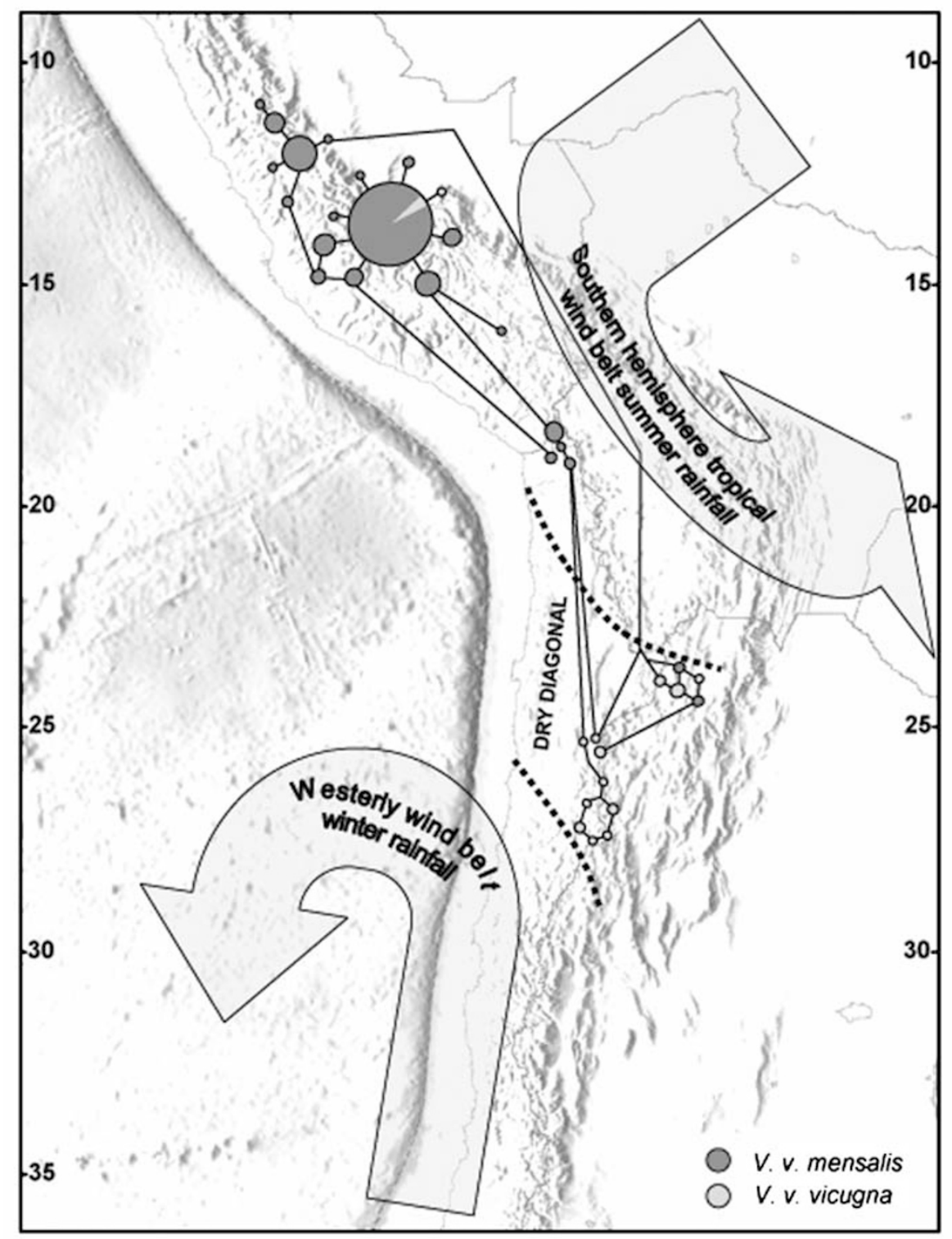

Figure 4 Map of Andean South America showing the position of the Dry Diagonal in relation to the Southern hemisphere tropical wind and Westerly wind belts.

AMOVA analyses also strongly support population groupings that correlate with currently defined subspecies $(50.49 \%$ of the variation) and geographic origin (ecotypes 'in' or 'out' of the Dry Diagonal; 38.43\%); however, little variance was explained by population subdivision within 'subspecies' $(9.94 \%)$ or by subdivision among countries $(13.31 \%)$. This finding contrasts with the results of Sarno et al. (2004), who reported no significant differentiation between subspecies, but again this could be owing to differences in sample sizes between the two data sets. The distinction between northern and southern populations is further evidenced by the phylogenetic tree and haplotype network (Figures 2 and 3, respectively), although reciprocal monophyly is not observed between subspecies. High divergence is seen between, but little structure is observed within, northern and southern groups, with a high degree of mixing of haplotypes from different locations.

\section{Demographic history}

There is strong molecular support for the validity of two groups in vicuña, with a larger proportion of the variance being explained by following current subspecies designation as opposed to ecotype. However, both values are significant and the two hypotheses are not mutually exclusive. Indeed, the patterns seen may not relate directly to divergence in isolation (vicariant speciation) or adaptive divergence, but may simply reflect the demographic history of the species as a whole, with incomplete lineage sorting or possibly resulting from rare dispersal events (e.g., Madison and Knowles, 2006; Tolley et al., 2006).

Consideration of the overall, high haplotype diversity, relatively low nucleotide diversity, negative $\mathrm{Fu}^{\prime} \mathrm{s} F_{\mathrm{S}}$ values and positive $g$ statistics indicate that the species as a whole has undergone a rapid demographic 
expansion. The presence of a large dominant haplotype (V3) possibly represents an ancestral lineage, since older haplotypes are expected to have a wider geographic range and frequency (Templeton et al., 1995) compared with those which are more recent (but see Paulo et al., 2002). This haplotype is predominant in the northern populations, whereas the distribution of southern haplotypes is more disparate. When the groups are considered separately, we observe subtly different signatures of population history. It is clear from the results in Table 3, the shallow phylogenetic tree, haplotype network and from mismatch distributions (not shown) that northern vicunas have expanded to a greater extent than those in the south, whose populations show much weaker signatures of demographic expansion. Population expansion is most likely to be consistent with a late Pleistocene recolonisation event.

The effects of climatic oscillations and their influence on the distribution of the premontane and montane grassland utilised by vicuña are likely to have had a significant effect on the distribution of vicuña populations and patterns of genetic diversity. In South America, the Pleistocene glaciation was milder and more restricted than in the northern hemisphere, occurring only in the Andes (Clapperton, 1993), where the Late Glacial stage began 19-14000 and ended 11-10000 YBP (Dillehay, 2000). In addition, Andean glaciation is correlated with the amount of available moisture rather than a fall in temperature (Clayton and Clapperton, 1997; Ammann et al., 2001; Kull et al., 2002), leading to palaeolake transgressions and an increase in suitable grassland habitat. Fossil remains of vicuña from dates before the Pleistocene/Holocene transition have only been found at palaeontological localities in the lowlands east of the Andes, where vicuña apparently evolved from Hemiauchenia approximately 2 million years ago (Wheeler, 1995). Archaeological excavations have produced a few vicuña remains at sites from Tierra del Fuego (Prieto and Canto, 1997), Patagonia (Nami and Menegaz, 1991) and northern Paraguay (Ubilla, 2004), reflecting previous widespread lowland distribution between 13000 and 10000 YBP. Nonetheless, the earliest high-elevation occupations in central Peru reflect massive presence of vicuña in the centre of the moist puna by 10000 YBP (Wheeler, 1995), indicating the expansion of this species into this area as documented by the DNA data. The presence of a Dry Diagonal belt between $24^{\circ}$ and $29^{\circ} \mathrm{S}$ (Ammann et al., 2001; Kull et al., 2002) delimits the present day distribution of the southern vicuña ecotype and it is possible that at the end of the Pleistocene period these dry-adapted vicuña populations surviving in refuges within the Dry Diagonal became contained, unable to expand beyond it limits. Increased precipitation to the north of the arid belt during the late Pleistocene was associated with an increase of available grassland (Ammann et al., 2001; Kull et al., 2002) and would have supported the expansion of populations in the north. The Dry Diagonal is likely to have prevented southern expansions of northern, moist puna-adapted forms and the northern expansion of southern, dry puna-adapted forms resulting in the two potentially ecotypic groups that are evident today.

\section{Implications for conservation}

The results from mtDNA analyses support the existence of a northern and southern vicuña taxa/ESU differing in morphological and genetic traits. Consideration of past climatic events suggests a long geographic separation of these two forms resulting in their contrasting demographic histories. Consequently, we suggest that these two subspecies should be managed separately to preserve their local adaptations. On these grounds, we predict that intermediate populations might exhibit lower fitness than those further north and south. Additional analyses at nuclear loci microsatellites will provide increased resolution on the genetic differentiation among these populations.

\section{Acknowledgements}

We gratefully acknowledge the financial support from the following organisations: CONICYT, Chile (Beca de Apoyo a Tesis Doctoral), Darwin Initiative for the Survival of Species (UK) grant 162/06/126, The British Embassy (Lima), NERC (UK) grant GST/02/828, European Commission INCO-DC ICA4-2000-10229, Sustainable economic utilization of wild South American camelids: strategies for improving rural productivity in pastoral communities in Latin America - MACS. In Chile we thank the Servicio Agrícola y Ganadero, SAG (Permit 447, 2002), the Corporación Nacional Forestal, CONAF (permit 6/02/2002) for granting other collection permits and help in collecting samples Cristian Bonacic (Facultad de Agronomía e Ingeniería Forestal, Pontificia Universidad Católica de Chile), Eduardo Palma (Departamento de Ecología and Center for Advanced Studies in Ecology and Biodiversity, PUC), Benito González (Facultad de Ciencias Veterinarias y Pecuarias, Universidad de Chile), Bibiana Vilá (Universidad de Lujan), Luis Jacome (Zoológico de Buenos Aires, Argentina) and Alberto Duarte (Zoológico de Mendoza, Argentina) for sharing samples. In Peru, special thanks go to Carlos Loret de Mola and Maria Luisa del Rio (CONAM); Wilder Trejo, Daniel Rivera, Daniel Arestegui, Leonidas Rodriguez, Carlos Flores, and Dirky Arias at CONACS; Gustavo Suarez de Freitas and Antonio Morizaki at INRENA; Felipe San Martin (Facultad de Medicina Veterinaria, Universidad Nacional Mayor de San Marcos) and Alejandro Camino (Asociacion Ancash). At CONOPA, Hugo Castillo, Lenin Maturrano and Rocio Quispe, have also made important contributions to this paper. Finally, we thank Iain Gordon and Jerry Laker (Macaulay Land Use Research Institute, MLURI) for bringing the Chilean and Peruvian partners together within the framework of the MACS project.

\section{References}

Ammann C, Jenny B, Kammer K, Messerli B (2001). Late Quaternary Glacier response to humidity changes in the arid Andes of Chile (18-29 $\left.9^{\circ}\right)$. Palaeogeogr Palaeoclimatol Palaeoecol 172: 313-326.

Bruford MW, Hanotte O, Brookfield JFY, Burke T (1998). Single and multilocus DNA fingerprinting. In: AR Hoelzel (ed). Molecular Genetic Analysis of Populations: A Practical Approach, 2nd edn. Oxford University Press: Oxford.

Clapperton CM (1993). The Quaternary Geology and Geomorphology of South America. Elsevier: Amsterdam. 
Clayton JD, Clapperton CM (1997). Broad synchrony of a late glacial glacier advance and the highstrand of paleolake Tauca in the Bolivian Altiplano. J Quaternary Sci 12: 159-182.

Clement M, Posada D, Crandall K (2000). TCS: a computer program to estimate gene genealogies. Mol Ecol 9: 1657-1659.

Dillehay TD (2000). The Settlement of the Americas. Basic Books, Perseus Book Group: New York.

Excoffier L (2004). Patterns of DNA sequence diversity and genetic structure after a range expansion: lessons from the infinite-island model. Mol Ecol 13: 853-864.

Excoffier L, Laval G, Schneider S (2005). Arlequin ver. 3.0: an integrated software package for population genetics data analysis. Evolutionary Bioinformatics Online 1: 47-50.

$\mathrm{Fu}$ YX (1996). New statistical tests of neutrality of mutations. Genetics 143: 557-570.

Grimwood IR (1969). Notes on the distribution and status of some Peruvian mammals 1968. American Committee for International Wildlife Protection. New York Zoological Society Special Publication No. 21: New York.

Harrison JA (1979). Revision of the Camelinae (Artiodactyla Tylopoda) and description of the new genus alforjas. Paleontol Contributions Univ Kansas 95: 1-20.

Hoffstetter R (1986). High Andean mammalian faunas during the Plio-Pleistocene. In: Vuilleumier F, Monasterio M (eds). High Altitude Tropical Biogeography. Oxford University Press: Oxford. pp 218-245.

Jungius $H$ (1971). The Vicuña in Bolivia: the status of an endangered species and recommendations for its conservation. Zeitschrift für Säugetierkunde 36: 129-146.

Kadwell M, Fernández M, Stanley HF, Baldi R, Wheeler JC, Rosadio R et al. (2001). Genetic analysis reveals the wild ancestors of the llama and alpaca. Proc Royal Soc London B 268: 2575-2584.

Koford CB (1957). The Vicuña and the Puna. Ecol Monogr 27: 153-219.

Kuhner MK, Yamato J, Felsenstein J (1998). Maximum likelihood estimation of population growth rates based on the coalescent. Genetics 149: 429-434.

Kull C, Grosjean M, Veit H (2002). Modeling modern and late Pleistocene glacio-climatological conditions in the North Chilean Andes (29-30 $\mathrm{S})$. Climatic Change 52: 359-381.

Lin Z, Nomura O, Hayashi T, Wada Y, Yasue H (2001). Characterization of a SINE species from vicuna and its distribution in animal species including the family Camelidae. Mamm Genome 12: 305-308.

MacFadden BJ, Siles O, Zeitler P, Johnson NM, Campbell KE. (1983). Magnetic polarity stratigraphy of the middle Pleistocene (Ensenadan) Tarija formation of Southern Bolivia. Quat Res 19: 172-187.

Madison WP, Knowles LL (2006). Inferring phylogeny despite incomplete lineage sorting. Syst Biol 55: 21-30.

Marín JC (2004) Filogenia molecular, filogeografía y domesticación de camelidos sudamericanos (ARTIODACTYLA: CAMELIDAE). PhD thesis, Universidad de Chile, Santiago.

Menegaz AN, Goin FJ, Ortiz Jaureguizar E (1989). Analisis morfologico y morfometrico multivariado de los representantes follies y viventes del genero Lama (Artiodactyla, Camelidae). Sus implicancias sistematicas, biogeograficas, ecologicas y biocronologicas. Ameghiniana 26: 153-172.

Maté ML, Di Rocco F, Zambelli A, Vidal-Rioja L (2004). Mitochondrial DNA structure and organization of the control region of South American camelids. Mol Ecol Notes 4: 765-767.

Miller Jr GS (1924). A second instance of the development of rodent-like incisors in an artiodactyl. Proc United States Natl Museum 66: 1-4.

Molina GI (1782). Saggio Sulla Storia Naturale del Chile. Bologna, Tommaso d'Aquino, p 367.

Nami HG, Menegaz AN (1991). Cueva del Medio: aportes para el conocimiento de la diversidad faunística hacia el Pleistoceno-Holoceno en la Patagonia austral. Anales Instituto Patagónico Serie Ciencies Historicas 20: 117-132.
Norambuena MC, Paredes M (2003). Variabilidad y estructura genetica en dos poblaciones de Vicugna vicugna (Camelidae) from northern Chile. Rev Chil Hist Nat 76: 99-104.

Palma RE, Marín JC, Spotorno AE, Galaz JL (2001). Phylogenetic relationships among south american subspecies of camelids based on secuences of cytochrome $b$ mitochondrial genes. In: Raunelli MGA (ed). EEAP publication No. 105, Göttingen, Germany 27-29 May 1999 Progress in South American Camelids Research. Wageningen Press: Göttingen, Alemania. pp 44-52.

Paulo OS, Jordan WC, Bruford MW, Nichols RA (2002). Using nested clade analysis to assess the history of colonization and the persistence of populations of an Iberian lizard. Mol Ecol 11: 809-819.

Posada D, Crandall KA (1998). MODELTEST: testing the model of DNA substitution. Bioinformatics 14: 817-818.

Prieto A, Canto J (1997). Presencia de un lamoide atipico en Cueva Lago Sofia 4 (Ultima Esperanza) y Tres Arroyos (Tierra del Fuego) Region de Magallanes, Chile. Anales Instituto Patagónico Serie Ciencies Historicas 25: 147-150.

Ray N, Currat M, Excoffier L (2003). Intra-deme molecular diversity in spatially expanding populations. Mol Biol Evol 20: $76-86$

Rozas J, Rozas R (1999). DnaSP version 3: an integrated program for molecular population genetics and molecular evolution analysis. Bioinformatics 15: 174-175.

Sarno RJ, Villalba L, Bonacic C, Gonzalez B, Zapata B, Mac Donald DW et al (2004). Phylogeography and subspecies assessment of vicunas in Chile and Bolivia utilizing mtDNA and microsatellite markers: implications for vicuna conservation and management. Conserv Genet 5: 89-102.

Schaschl H, Kaulfus D, Hammer S, Suchentrunk F (2003). Spatial patterns of mitochondrial and nuclear gene pools in chamois (Rupicapra r. rupicapra) from the Eastern Alps. Heredity 91: 125-135.

Schneider S, Roessli D, Excoffier L (2000). Arlequin ver. 2000: A Software for Population Genetics Data Analysis. Genetics and Biomety Laboratory: University of Geneva, Switzerland.

Sharma P, Elmore D, Dunne A, DeSilva SL, Vogt S (1995). Application of $35 \mathrm{Cl}$ surface exposure age dating to Central Andean glaciology and volcanology. American Geophysical Union Fall Meeting 2005, Abstracts.

Stanley HF, Kadwell M, Wheeler JC (1994). Molecular evolution and genetic diversity of the Camelidae. Proc Royal Soc London $B$ 256: 1-6.

Swofford DL (2001). PAUP*. Phylogenetic analysis using parsimony (* and other methods). Version 4. Sinauer Associates: Sunderland, MA.

Tajima F (1989). Statistical method for testing the neutral mutation hypothesis by DNA polymorphism. Genetics 123 : 585-595.

Templeton AR, Routman E, Phillips CA (1995). Separating population structure from population history: a cladistic analysis of the geographical distribution of mitochondrial DNA haplotypes in the tiger salamander, Ambystoma tigrinum. Genetics 140: 767-782.

Thomas O (1917). Preliminary diagnosis of new mammals obtained by the Yale National Society Peruvian Expedition. Smithsonian Misc C 68: 1-3.

Thornback J, Jenkins M (eds). (1982). The IUCN Mammal Red Data Book. Unwin Brothers - Greshman Press: Surrey.

Tolley KA, Burger M, Turner AA, Matthee CA (2006). Biogeographic patterns and phylogeography of dwarf chameleons (Bradypodion) in an African biodiversity hotspot. Mol Ecol 15: 781-793.

Tserenbataa T, Ramey RR, Ryder OA, Quinn TW, Reading RP (2004). A population genetic comparison of argali sheep (Ovis ammon) in Mongolia using the ND5 gene of 
mitochondrial DNA; implications for conservation. Molr Ecol 13: 1333-1339.

Ubilla M (2004). Mammalian biostratigraphy of Pleistocene fluvial deposits in northern Uruguay, South America. Proc Geologist Assoc 115: 347-357.

Watterson GA (1975). On the number of segregating sites. Theor Popul Biol 7: 256-276.

Webb SD (1974). Pleistocene Llamas of Florida, with a brief review of the Lamini. In: Webb SD (ed). Pleistocene Mammals of Florida. The University Presses of Florida: Gainesville, pp 170-213.
Wheeler JC (1995). Evolution and present situation of the South American Camelidae. Biol J Linn Soc 54: 271-295.

Wheeler JC (2006). Historia Natural de la Vicuña. In: Vilá B (ed). Investigacion Conservacion y Manejo de Vicuñas. Proyecto MACS: Buenos Aires, Argentina. pp 25-36.

Wheeler JC, Hoces Roque D (1997). Community participation, sustainable use and vicuña conservation in Peru. Mt Res Dev 17: 283-287.

Wheeler JC, Pires-Ferreira E, Kaulicke P (1976). Preceramic animal utilization in the Central Peruvian Andes. Science 194: 483-490. 